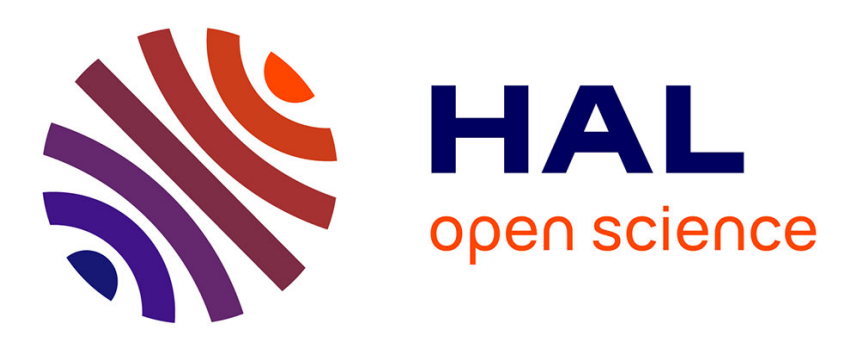

\title{
Robust Adaptive Control of Automatic Guidance of Farm Vehicles in the Presence of Sliding
} Hao Fang, Romain Lenain, Benoît Thuilot, Philippe Martinet

\section{To cite this version:}

Hao Fang, Romain Lenain, Benoît Thuilot, Philippe Martinet. Robust Adaptive Control of Automatic Guidance of Farm Vehicles in the Presence of Sliding. 2005 IEEE International Conference on Robotics and Automation, Apr 2005, Barcelona, Spain. pp.3102-3107, 10.1109/ROBOT.2005.1570587 . hal02467172

\section{HAL Id: hal-02467172 \\ https://hal.inria.fr/hal-02467172}

Submitted on 5 Feb 2020

HAL is a multi-disciplinary open access archive for the deposit and dissemination of scientific research documents, whether they are published or not. The documents may come from teaching and research institutions in France or abroad, or from public or private research centers.
L'archive ouverte pluridisciplinaire HAL, est destinée au dépôt et à la diffusion de documents scientifiques de niveau recherche, publiés ou non, émanant des établissements d'enseignement et de recherche français ou étrangers, des laboratoires publics ou privés. 


\title{
Robust Adaptive Control of Automatic Guidance of Farm Vehicles in the Presence of Sliding
}

\author{
H. Fang, R. Lenain, B. Thuilot and P. Martinet \\ LASMEA \\ 24, av. des Landais \\ 63177 Aubiere Cedex France \\ hao@lasmea.univ-bpclermont.fr
}

\begin{abstract}
High-precision autofarming is rapidly becoming a reality with the requirements of agricultural applications. Lots of research works have been focused on the automatic guidance control of farm vehicles, satisfactory results have been reported under the assumption that vehicles move without sliding. But unfortunately the pure rolling constraints are not always satisfied especially in agriculture applications where the working conditions are rough and not expectable. In this paper the problem of path following control of autonomous farm vehicles in presence of sliding is addressed. To take sliding effects into account, a vehicle-oriented kinematic model is constructed in which sliding effects are introduced as additive unknown parameters of the ideal kinematic model. Based on backstepping method a stepwise procedure is proposed to design an adaptive controller in which timeinvariant sliding effects are learned and compensated by parameter adaptations. It is theoretically proven that for the farm vehicles subject to sliding, the lateral deviation can be stabilized near zero and the orientation errors converge into a neighborhood near the origin. To be more robust to disturbances including external noises and unmodeled time-varying sliding components, the adaptive controller is refined by integrating Variable Structure Controllers (VSC) or projection mappings. Simulation results show that the proposed robust adaptive controllers can reject sliding effects and guarantee high path-following accuracy.
\end{abstract}

Index Terms-Robust Adaptive Control, Nonholonomic Systems, Backstepping Control

\section{INTRODUCTION}

High-precision auto-farming and precise automatic guidance of agricultural vehicles have been the subject of research for a long time. Lots of satisfactory results of path following control have been achieved provided vehicles satisfy the pure rolling constraints [1]-[3]. In our previous works we have solved the problem of curved path following of farm vehicles with unique RTK GPS [6], the ideal vehicle kinematic model is constructed, a nonlinear controller guaranteeing high lateral and orientation accuracy has been designed by converting vehicle kinematic model into a chained system.

However due to various effects such as slipping of tires, deformability or flexibility of wheels, the pure rolling constraints are never strictly satisfied. Especially in agriculture applications when farm vehicles are required to move on all-terrain grounds including slippery slopes, sloppy grass grounds, sandy and stony grounds, where contact conditions between wheels and grounds are unexpectable and change greatly, sliding inevitably occurs which deteriorates performances of automatic guidance and even system stability.

Until now there are very few papers dealing with sliding. [8] prevents cars from skidding by robust decoupling of car steering dynamics which is achieved by feedback of the integrated yaw rate into front wheel steering, but acceleration measurements are necessary and the steering angle is assumed small. [9] copes with the control of WMR (Wheeled Mobile Robot) not satisfying the ideal kinematic constraints by using slow manifold methods, but the parameter characterizing the sliding effects is assumed to be exactly known. Therefore [8][9] are not realistic for agriculture applications. In [10] a controller is designed based on the averaged model allowing the tracking errors to converge to a limit cycle near the origin. In [14] a general singular perturbation formulation is developed which lead to robust results for linearizing feedback laws ensuring trajectory tracking. But above two schemes only take into account sufficiently small skidding effects and they are too complicated for real-time practical implementation. In [11] [12] variable structure control is used to eliminate the harmful sliding effects when the bounds of the sliding effects have been known. The trajectory tracking problem of mobile robots in the presence of sliding is solved in [13] using discrete-time sliding mode control which is robust against the sliding effects. But the controllers [11][13] counteract sliding effects only relying on high-gain controllers which is not realistic because of low level delay introduced by steering systems of farm vehicles. In [15] the sliding effects are rejected by re-scheming the desired path adaptively based on the steady control errors which are mainly caused by the modeled sliding effects, the actual implementation has validated its effectiveness but the proof of convergence is still open.

In above references most research works treated sliding as disturbance, but alternatively sliding can be also regarded specifically as time-varying parameters. On the other hand backstepping methods which have been used widely in controller design are proven powerful in controlling nonholonomic systems with uncertain parameters [16][18], so the purpose of this paper is to use backstepping methods to design a practical path following controller in presence of sliding with small control gains. It has been well known that vehicle positions and velocities cannot 
be measured by relative localization systems for example incremental encoders when sliding occurs, so absolute localization is necessary for anti-sliding control. The absolute localization technologies include active beacon, GPS, laser range scans and multi-sensor data fusion etc. In addition, with the development of GPS technology more and more researchers apply GPS to automatic guidance systems of agricultural vehicles [4]-[7], since GPS can provide realtime absolute positions with centimeter accuracy and outdoor working environments of agricultural vehicles are suitable for using GPS. Due to those two points and our previous works [6], in this paper the problem of anti-sliding controller design is addressed for the farm vehicles with GPS localization systems.

The main idea of this paper is to regard sliding effects as unknown parameters added to the ideal kinematic model. Based on backstepping method an adaptive controller is designed to estimate and compensate modeled time-invariant sliding components. Furthermore two approaches are proposed to refine the adaptive controller, making it more robust to the time-varying sliding effects and disturbances. This paper is organized as follows, in section II a vehicleoriented kinematic model considering sliding is constructed in the path frame. In section III by assuming sliding is timeinvariant, an adaptive controller is designed using backstepping methods. In section IV robust adaptive controllers are obtained by integrating VSC or applying projection mapping. In section $\mathrm{V}$ some comparative simulation results are presented to validate the proposed control law.

\section{KINEMATIC MODEL}

\section{A. Notation and Problem Description}

In this paper the vehicle is simplified into a bicycle model. The kinematic model is expressed with respect to the path in frame $\left(M, \eta_{t}, \eta_{n}\right)$, variables necessary in the kinematic model are denoted as follows: (see figure 1)

- $C$ is the path to be followed.

- $O$ is the center of the vehicle virtual rear wheel.

- $M$ is the orthogonal projection of $O$ on path $C$.

- $\eta_{t}$ is the tangent vector to the path at $M$.

- $\eta_{n}$ is the normal vector at $M$.

- $y$ is the lateral deviation between $O$ and $M$

- $s$ is the curvilinear coordinates (arc-length) of point $M$ along the path from an initial position.

- $c(s)$ is the curvature of the path at point $M$.

- $\theta_{d}(s)$ is the orientation of the tangent to the path at point $M$ with respect to the inertia frame.

- $\theta$ is the orientation of the vehicle centerline with respect to the inertia frame.

- $\tilde{\theta}=\theta-\theta_{d}$ is the orientation error.

- $l$ is the vehicle wheelbase.

- $v$ is the vehicle linear velocity.

- $\delta$ is the steering angle of the virtual front wheel

So the vehicle movement can be described by $(y, s, \tilde{\theta})$. In this paper path following control law

$$
\delta=K(s, y, \tilde{\theta}, v)
$$

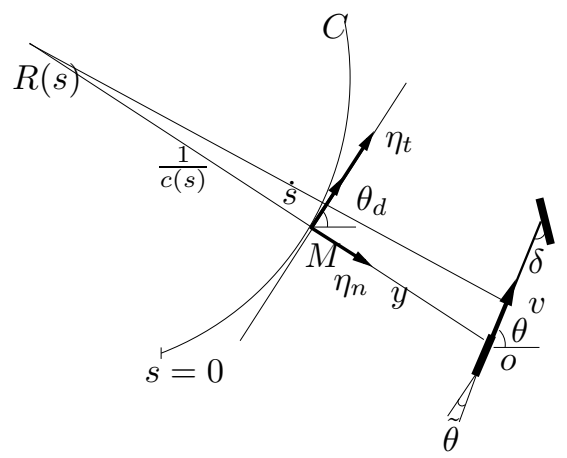

Fig. 1. Notation and path frame description

will be designed to guarantee

$$
\lim _{t \rightarrow \infty} y=0
$$

and $\tilde{\theta}$ is bounded in presence of sliding.

\section{B. Kinematic Model}

When the vehicles move without sliding, the ideal kinematic model of the vehicles is (see [6]).

$$
\left\{\begin{aligned}
\dot{s} & =\frac{v \cos \tilde{\theta}}{1-c(s) y} \\
\dot{y} & =v \sin \tilde{\theta} \\
\dot{\tilde{\theta}} & =v\left(\frac{\tan \delta}{l}-\frac{c(s) \cos \tilde{\theta}}{1-c(s) y}\right)
\end{aligned}\right.
$$

But when the vehicles move on a steep slope or the ground is slippery, sliding occurs inevitably, (3) is no longer valid. The violation of the pure rolling constraints is described by introducing the lateral sliding velocity $v_{y}$ and steering angle bias $\delta_{b}$ (figure 2). Similar developing methods lead to

$$
\left\{\begin{aligned}
\dot{s} & =\frac{v \cos \tilde{\theta}}{1-c(s) y}-\frac{v_{y} \sin \tilde{\theta}}{1-c(s) y} \\
\dot{y} & =v \sin \tilde{\theta}+v_{y} \cos \tilde{\theta} \\
\dot{\tilde{\theta}} & =v\left(\frac{\tan \left(\delta+\delta_{b}\right)}{l}-\frac{c(s) \cos \tilde{\theta}}{1-c(s) y}\right)-\frac{v_{y}}{l}+\frac{c(s) v_{y} \sin \tilde{\theta}}{1-c(s) y}
\end{aligned}\right.
$$

Since $\delta_{b}$ is quite small, a linearized kinematic model can be obtained

$$
\left\{\begin{array}{l}
\dot{y}=v \sin \tilde{\theta}+\psi(t) \cos \tilde{\theta} \\
\dot{\tilde{\theta}}=v\left(\frac{\tan \delta}{l}-\frac{c(s) \cos \tilde{\theta}}{1-c(s) y}\right)+\frac{v}{l} \eta(t)+\left(\frac{c(s) \sin \tilde{\theta}}{1-c(s) y}-\frac{1}{l}\right) \psi(t)
\end{array}\right.
$$

where $\psi(t)=v_{y}, \eta(t)$ is linked to $\delta_{b}$ including inaccuracy due to linearization approximation.

\section{Adaptive Control Law Design}

In actual agriculture applications, farm vehicles always move with smooth velocities and most paths to be followed are straight lines and circles. The accelerations and the path curvature vary quite slowly with time, hence $\psi(t), \eta(t)$ can be approximated as

$$
\left\{\begin{array}{l}
\psi(t)=\psi_{s}+\breve{\psi}(t) \\
\eta(t)=\eta_{s}+\breve{\eta}(t)
\end{array}\right.
$$




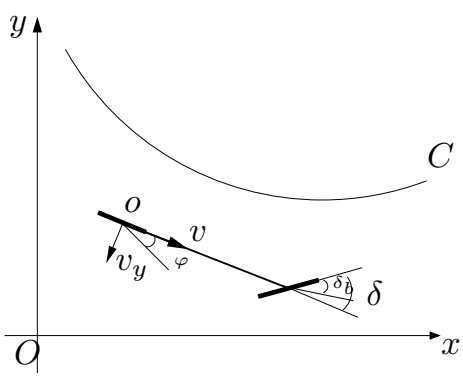

Fig. 2. Notations of sliding effects

where the sliding components $\psi_{s}, \eta_{s}$ are almost timeinvariant, $\breve{\psi}(t), \breve{\eta}(t)$ are time-varying variables. In this section by assuming $\dot{\psi}_{s}=\dot{\eta}_{s}=0$ and $\breve{\psi}(t)=\breve{\eta}(t)=0$, an adaptive controller will be designed. The inaccuracy of this approximation mainly consisting of trivial timevarying sliding effects is not important, it will be treated as disturbances and rejected by robust controller design in forthcoming section IV.

\section{A. Backstepping-based Control Design Scheme}

In the model (5), the lateral deviation $y$ is not directly controlled. To overcome this problem the idea of backstepping is used; see [17] for details. Using backstepping we propose a stepwise control design procedure for this 2-order nonholonomic system with unknown parameters.

step 1: Consider the lateral subsystem of (5) and assumption of time-invariant sliding, the Lyapunov function candidate is chosen as

$$
V_{1}=\frac{1}{2} y^{2}+\frac{1}{2}\left(\hat{\psi}_{s}-\psi_{s}\right)^{T} \Gamma^{-1}\left(\hat{\psi}_{s}-\psi_{s}\right)
$$

where $\Gamma$ is positive definite, $\hat{\psi}_{s}$ indicates the estimation of $\psi_{s}$. The time derivative of $V_{1}$ along the kinematic model is

$$
\dot{V}_{1}=y\left(v \sin \tilde{\theta}+\hat{\psi}_{s} \cos \tilde{\theta}\right)+\left(\hat{\psi}_{s}-\psi_{s}\right)^{T} \Gamma^{-1}\left(\dot{\hat{\psi}}_{s}-\Gamma y \cos \tilde{\theta}\right)
$$

Regard $u_{1}=\sin \tilde{\theta}$ as the virtual control input of the first step. If choose $u_{1}$ as

$$
u_{1 d}=\frac{-k_{1} y-\hat{\psi}_{s} \cos \tilde{\theta}}{v}
$$

and

$$
\dot{\hat{\psi}}_{s}=\Gamma y \cos \tilde{\theta}
$$

then we have

$$
\dot{V}_{1}=-k_{1} y^{2}
$$

$u_{1 d}$ is the desired value of the virtual control input $u_{1}$ for the first step. If $u_{1}$ tracks (9) precisely, then the lateral subsystem of (5) can be stabilized asymptotically.

In the closed-loop system $u_{1}$ is not the actual control input, tracing $u_{1 d}$ with some errors indeed, so an error variable $\tilde{u}_{1}$ is defined as

$$
\tilde{u}_{1}=u_{1}-u_{1 d}
$$

The time derivative of $\tilde{u}_{1}$ can be computed as

$$
\begin{aligned}
& \dot{\tilde{u}}_{1}=m_{1}\left(v\left(\frac{\tan \delta}{l}-\frac{c(s) \cos \tilde{\theta}}{1-c(s) y}\right)+m_{3} \eta_{s}+m_{2} \psi_{s}\right) \\
& +\frac{\dot{\hat{\psi}}_{s} \cos \tilde{\theta}+k_{1}\left(v \sin \tilde{\theta}+\psi_{s} \cos \tilde{\theta}\right)}{v}
\end{aligned}
$$

where

$$
\begin{aligned}
& m_{1}=\cos \tilde{\theta}-\frac{\hat{\psi}_{s} \sin \tilde{\theta}}{v} \\
& m_{2}=\frac{c(s) \sin \tilde{\theta}}{1-c(s) y}-\frac{1}{l} \\
& m_{3}=\frac{v}{l}
\end{aligned}
$$

Note that

$$
m_{1}=\frac{1}{\cos \hat{\varphi}} \cos (\tilde{\theta}+\hat{\varphi})
$$

where $\hat{\varphi}$ is the estimation of the slip angle defined in the figure 2. By assuming $|\tilde{\theta}+\hat{\varphi}|<\frac{\pi}{2}$, one get $m_{1}>0$.

Remark: For simplicity it is assumed that $v$ is constant, in case $v$ is time-varying, only variation is adding $\frac{\left(-k_{1} y-\hat{\psi}_{s} \cos \tilde{\theta}\right) \dot{v}}{v^{2}}$ in (13).

step 2: Considering the Lyapunov function as

$$
V_{2}=V_{1}+\frac{1}{2} \tilde{u}_{1}^{2}+\frac{1}{2}\left(\hat{\eta}_{s}-\eta_{s}\right)^{T} \gamma^{-1}\left(\hat{\eta}_{s}-\eta_{s}\right)
$$

where $\gamma$ is positive definite, $\hat{\eta}_{s}$ indicates the estimation of $\eta_{s}$. Regard $u_{2}=\tan \delta$ as the virtual control input of the second step, then the time derivative of $V_{2}$ along (8)(13) is

$$
\begin{aligned}
& \dot{V}_{2}=y\left(v u_{1}+\hat{\psi}_{s} \cos \tilde{\theta}\right)+\left(\hat{\psi}_{s}-\psi_{s}\right)^{T} \Gamma^{-1}\left(\dot{\hat{\psi}}_{s}-\Gamma y \cos \tilde{\theta}\right) \\
& +\tilde{u}_{1} m_{1}\left(v\left(\frac{u_{2}}{l}-\frac{c(s) \cos \tilde{\theta}}{1-c(s) y}\right)+m_{3} \eta_{s}+m_{2} \psi_{s}\right) \\
& +\tilde{u}_{1} \frac{k_{1}\left(v \sin \tilde{\theta}+\psi_{s} \cos \tilde{\theta}\right)+\dot{\hat{\psi}}_{s} \cos \tilde{\theta}}{v}+\left(\hat{\eta}_{s}-\eta_{s}\right)^{T} \gamma^{-1} \dot{\hat{\eta}}_{s}
\end{aligned}
$$

Substituting (9)(12) into (17), we have the following equation (see [23] for detail)

$$
\begin{aligned}
& \dot{V}_{2}=-k_{1} y^{2}+\tilde{u}_{1} m_{1}\left(v\left(\frac{u_{2}}{l}-\frac{c(s) \cos \tilde{\theta}}{1-c(s) y}\right)+m_{3} \hat{\eta}_{s}+m_{2} \hat{\psi}_{s}\right) \\
& +y v \tilde{u}_{1}+\tilde{u}_{1} \frac{k_{1}\left(v \sin \tilde{\theta}+\hat{\psi}_{s} \cos \tilde{\theta}\right)+\hat{\psi}_{s} \cos \tilde{\theta}}{v} \\
& +\left(\hat{\eta}_{s}-\eta_{s}\right)^{T} \gamma^{-1}\left(\dot{\hat{\eta}}_{s}-\tilde{u}_{1} \gamma m_{1} m_{3}\right) \\
& +\left(\hat{\psi}_{s}-\psi_{s}\right)^{T} \Gamma^{-1}\left(\dot{\hat{\psi}}_{s}-\Gamma y \cos \tilde{\theta}-\Gamma k_{1} \frac{\tilde{u}_{1} \cos \tilde{\theta}}{v}-\Gamma \tilde{u}_{1} m_{1} m_{2}\right)
\end{aligned}
$$

In (18) let

$$
\begin{aligned}
& \dot{\hat{\psi}}_{s}=\Gamma y \cos \tilde{\theta}+\frac{\Gamma k_{1} \cos \tilde{\theta}}{v} \tilde{u}_{1}+\Gamma \tilde{u}_{1} m_{1} m_{2} \\
& \dot{\hat{\eta}}_{s}=\gamma m_{1} m_{3} \tilde{u}_{1}
\end{aligned}
$$

and choose $u_{2}$ as

$$
u_{2}=l\left(-\frac{y}{m_{1}}-\frac{k_{2} \tilde{u}_{1}}{m_{1} v}-\frac{m_{3} \hat{\eta}_{s}+m_{2} \hat{\psi}_{s}}{v}+\alpha+\beta\right)
$$

where

$$
\alpha=-\frac{k_{1}\left(v \sin \tilde{\theta}+\hat{\psi}_{s} \cos \tilde{\theta}\right)+\dot{\hat{\psi}}_{s} \cos \tilde{\theta}}{v^{2} m_{1}}
$$




$$
\beta=\frac{c(s) \cos \tilde{\theta}}{1-y c(s)}
$$

then we get

$$
\dot{V}_{2}=-k_{1} y^{2}-k_{2} \tilde{u}_{1}^{2}
$$

Finally the vehicle steering angle $\delta$ is determined by

$$
\delta=\arctan u_{2}
$$

\section{B. Stability Analysis}

From (23) it is known that the solutions $y, \tilde{u}_{1}$ and estimation errors $\tilde{\psi}_{s}, \tilde{\eta}_{s}$ are bounded on $[0,+\infty)$. The direct application of LaSalle invariance principle yields that all the solutions converge to the set $\Omega$ with

$$
\Omega=\left\{\left(y, \tilde{u}_{1}, \hat{\psi}_{s}, \hat{\eta}_{s}\right): y=0, \tilde{u}_{1}=0\right\}
$$

From (25), one gets that the lateral deviation will converge to zero asymptotically, simultaneously the steady orientation error $\tilde{\theta}$ is bounded by

$$
\tan \tilde{\theta}=-\frac{\hat{\psi}_{s}}{v}
$$

It is obvious that when vehicles move without sliding $\left(\hat{\psi}_{s}=0\right)$, the orientation error will converge to zero.

\section{Robust Adaptive Controller Design}

\section{A. Kinematic Model with Time-varying Sliding}

We have designed an adaptive controller when assuming that the sliding effects are time-invariant, but from (5)(6) the precise kinematic model with time-varying sliding is

$$
\left\{\begin{array}{l}
\dot{y}=v \sin \tilde{\theta}+\psi_{s} \cos \tilde{\theta}+\varepsilon_{1} \\
\dot{\tilde{\theta}}=v\left(\frac{\tan \delta}{l}-\frac{c(s) \cos \tilde{\theta}}{1-c(s) y}\right)+m_{3} \eta_{s}+m_{2} \psi_{s}+\varepsilon_{2}
\end{array}\right.
$$

where

$$
\begin{aligned}
& \varepsilon_{1}=\breve{\psi}(t) \cos \tilde{\theta}+\varsigma_{1} \\
& \varepsilon_{2}=m_{3} \breve{\eta}(t)+m_{2} \breve{\psi}(t)+\varsigma_{2}
\end{aligned}
$$

$\varsigma_{1}, \varsigma_{2}$ are unknown external disturbances. It is still an open problem to design controllers using backstepping algorithm for a system with time-varying parameters in presence of bounded disturbances and noise. In the latest literature [18] a new parametrization and filter structure that take into account parameter variation lead to a new backstepping controller when the time variation of the parameters is known. It is indicated that the uncertainty in the parameters can be counteracted by increasing the values of the design parameters. But high-gain controllers are not realistic for farm vehicles because of constrained control inputs and limited bandwidth of the steering systems.

In agriculture implementations the time-varying sliding components $\breve{\psi}(t), \breve{\eta}(t)$ have small amplitudes and we also assume that external disturbances are bounded, so it is reasonable to assume that $\varepsilon_{1}, \varepsilon_{2}$ are bounded

$$
\left|\varepsilon_{i}\right|<\rho_{i}
$$

Using the same backstepping procedures as section III-A, we can prove that in the first step

$$
\begin{aligned}
\dot{V}_{1}= & y\left(v \sin \tilde{\theta}+\hat{\psi}_{s} \cos \tilde{\theta}\right)+\left(\hat{\psi}_{s}-\psi_{s}\right)^{T} \Gamma^{-1}\left(\dot{\hat{\psi}}_{s}-\Gamma y \cos \tilde{\theta}\right) \\
& +y \varepsilon_{1}
\end{aligned}
$$

Moreover applying the resulting adaptive laws (19) and the controller (20), we can prove that

$$
\dot{V}_{2}=-k_{1} y^{2}-k_{2} \tilde{u}_{1}^{2}+y \varepsilon_{1}+\tilde{u}_{1}\left(m_{1} \varepsilon_{2}+\frac{k_{1} \varepsilon_{1}}{v}\right)
$$

which implies that the closed-loop system is globally uniformly bounded.

\section{B. Robust Adaptive Controller with Variable Structure Controller}

Here we are in the place to design a controller which is robust to $\varepsilon_{i}$. Combining classical robust controllers with adaptive controllers is straightforward [19]. So in the first step we design $u_{1 d}$ as a variable structure controller from (30). Furthermore $\operatorname{sign}(\cdot)$ is replaced by $\tanh (\cdot)$ in this paper which is continuously differentiable in the stepwise procedures.

$$
u_{1 d}=\frac{-k_{1} y-\hat{\psi}_{s} \cos \tilde{\theta}-\rho_{1} \tanh \left(\frac{y}{\sigma_{3}}\right)}{v}
$$

Then following the similar stepwise procedures of section III-A, we get a robust adaptive controller

$$
u_{2}=l\left(-\frac{y}{m_{1}}-\frac{k_{2} \tilde{u}_{1}}{m_{1} v}-\frac{m_{3} \hat{\eta}_{s}+m_{2} \hat{\psi}_{s}}{v}+\alpha+\beta+s_{1}+s_{2}\right)
$$

where $\beta, \dot{\hat{\eta}}_{s}$ are defined as before and

$$
\begin{aligned}
& \alpha=-\frac{\varpi\left(v \sin \tilde{\theta}+\hat{\psi}_{s} \cos \tilde{\theta}\right)+\dot{\hat{\psi}}_{s} \cos \tilde{\theta}}{v^{2} m_{1}} \\
& s_{1}=-\frac{\rho_{1} \varpi}{v^{2} m_{1}} \tanh \left(\frac{\tilde{u}_{1}}{\sigma_{1}}\right) \\
& s_{2}=-\frac{\rho_{2}}{v} \tanh \left(\frac{\tilde{u}_{1}}{\sigma_{2}}\right) \\
& \dot{\hat{\psi}}_{s}=\Gamma y \cos \tilde{\theta}+\frac{\Gamma \varpi \cos \tilde{\theta}}{v} \tilde{u}_{1}+\Gamma \tilde{u}_{1} m_{1} m_{2} \\
& \varpi=k_{1}+\left(1-\tanh ^{2}\left(\frac{y}{\sigma_{3}}\right)\right) \frac{\rho_{1}}{\sigma_{3}}
\end{aligned}
$$

where $\sigma_{i}>0, \varpi$ is related to the derivative of $\tanh (), s_{1}$ is developed to reject $\varepsilon_{1}$ appearing in the derivative of $\tilde{u}_{1}$, $s_{2}$ is to reject $\varepsilon_{2}$ in $\dot{\tilde{\theta}}$. The controller (33) can guarantee the closed-loop system converge to $\Omega$. (see [23] for detail)

\section{Projection Mapping for Parameter Adaptation}

However the controller (33) has some drawbacks. It needs a large amount of on-board computation and highorder derivative of sign-like functions may lead to oscillating control inputs.

Another approach to design robust adaptive controllers is utilizing projection mapping for the parameter adaptation procedures. The projection mapping $\operatorname{Proj}_{\xi}(\bullet)$ is defined by [20], [21]

$$
\operatorname{Proj}_{\xi}(\bullet)= \begin{cases}0 & \text { if } \hat{\xi}=\xi_{\text {max }} \text { and } \bullet>0 \\ 0 & \text { if } \hat{\xi}=\xi_{\text {min }} \text { and } \bullet<0 \\ \bullet & \text { otherwise }\end{cases}
$$


By using projection mapping $\operatorname{Proj}_{\xi}(\bullet)$, the robust adaptive laws become

$$
\begin{gathered}
\dot{\hat{\psi}}_{s}=\operatorname{Proj}_{\psi_{s}}\left(\Gamma\left[\cos \tilde{\theta} \frac{k_{1} \cos \tilde{\theta}}{v}+m_{1} m_{2}\right]\left[\begin{array}{cc}
y & \tilde{u}_{1}
\end{array}\right]^{T}\right) \\
\dot{\hat{\eta}}_{s}=\operatorname{Proj}_{\eta_{s}}\left(\gamma m_{1} m_{3} \tilde{u}_{1}\right)
\end{gathered}
$$

The system will converge into a neighborhood of zero. (36)(37) can guarantee the estimation of sliding effects stay within a defined range regardless of external disturbances. The prior information on the bounds of the sliding effects $\psi_{s}, \eta_{s}$ can be obtained off-line after performing large number of absolute coordinates measurements under different typical working conditions.

\section{Simulation Results}

First a classical "U" path with a perfect circular arc (path $\# 1)$ is followed to test the adaptive controller. In the simulations, the gains used in (19), (20-21) are set as $k_{1}=0.15$, $k_{2}=1.14, \Gamma=0.15, \gamma=0.02$. In actual implementations, the gains should be tuned gradually to make an optimal compromise between transient characteristic and limited bandwidth of the steering system. The constant sliding is introduced with $v_{y}=-0.1, \delta_{b}=-0.048$. The control law in our previous work [6] is applied also with the controller gains $k_{p}=0.09, k_{d}=0.6$. Because this control law does not take sliding effects into account, in figure 3 when the sliding appears the lateral deviation (dashed line) becomes significant. While the controller (20) can compensate sliding effects through estimating them on line, the lateral deviation (solid line) converges to zero with small offsets (due to linearization approximation in (5)) after deviating from the desired path. Since small control gains are used, the vehicle movements are kept smooth. The remarkable overshoots at the beginning and end of the curve are caused by "jump change" of the sliding effects and low level delay. The robust adaptive controller with VSC (33) is simulated also. Because the modeling inaccuracy is counteracted by VSC, the lateral deviation can converge to zero with a good transient response (dotted line in figure 3 ). The bounded orientation errors are shown by figure 4 . As analyzed by section III-B the orientation errors do not converge to zero, indeed they are bounded by (26), it is normal when sliding occurs known as "crab sliding". The evolution of the sliding parameters $\psi$ (solid line), $\eta$ (dashed line) is shown by figure 5. It is clear that at the beginning and end of the circle, $\hat{\psi}$ varies greatly which explains the overshoots of the lateral deviation, but as the vehicle follows the circle, $\hat{\psi}, \hat{\eta}$ evolve smoothly close to the real values. To simulate the actual working condition, a set of real measurement data is used in the simulation to reconstruct the actual $v_{y}$ and $\delta_{b}$. In figure 6 the (robust) adaptive controllers yield small lateral deviation with zero mean value, while the lateral deviation of the controller [6] is significant and has obvious bias.

In order to compare the performances between the robust controllers (33) and projection mappings (36-37), another reference path \#2 consisting of straight lines and curves is followed (see figure 7), the lateral deviation is shown by figure 8 . The experimental data indicates that the robust controller with VSC yields better transient performances at the expense of non-smooth movements (dotted line) especially when low level delay is considered. While the controller with projection mapping yields a movement with less oscillation following the reference path (solid line). So for the vehicles with good low-level characters, the controller with VSC is favorable, but for the vehicles whose bandwidth is limited, the robust controller with projection mapping is preferred.

\section{CONCLUSION}

The path following problem of autonomous agricultural vehicles in the presence of sliding is investigated in this paper. A vehicle-oriented kinematic model which integrates the sliding effects as additive unknown parameters is constructed. From this model, a practical adaptive controller is designed based on the backstepping method which can stabilize the lateral derivation near zero and guarantees the orientation error converge into a neighborhood near the origin. In addition two approaches are proposed to refine the adaptive controller, allowing it to be robust to the time-varying sliding components and external disturbances. Experimental comparative results show the effectiveness of the proposed control laws. The advantages of this scheme are that

- When no sliding occurs, it provides a path following controller which can guarantee lateral deviation and orientation error converge to zero.

- Instead of using high gains or powerful functions to counteract sliding effects, small controller gains are used to stabilize the system and the sliding effects are

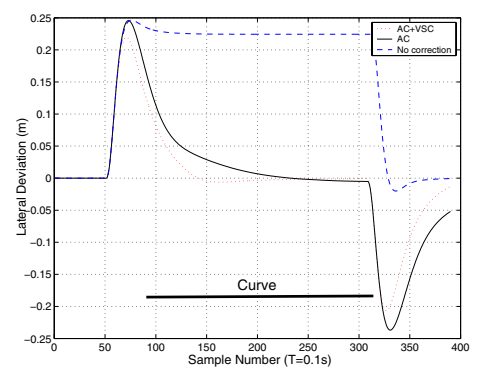

Fig. 3. Lateral deviation of path \#1

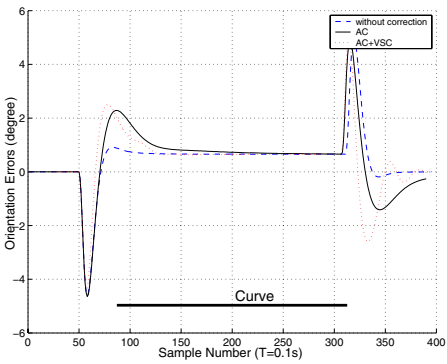

Fig. 4. Orientation errors of path \#1 


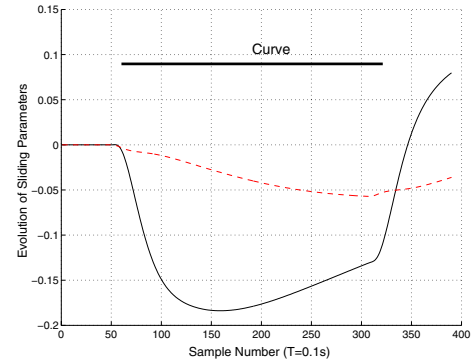

Fig. 5. Evolution of sliding parameters

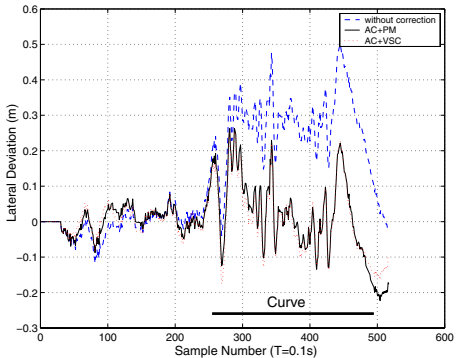

Fig. 6. Lateral deviation of path \#1 with real measurements

estimated by parameter adaptation, yielding precise path following with less oscillation.

- Backstepping procedures are used to design a pathfollowing controller for a 2-order nonholonomic system, it can be extended easily to high order nonholonomic systems.

The prospective works include extending backstepping methods to lateral-longitudinal control and using predictive control to decrease overshoots of lateral deviation [22].

\section{REFERENCES}

[1] Stombaugh, T. S. , “ Automatic Guidance of Agricultural Vehicles at Higher Speeds", Ph.D. dissertation. Dept. of Agriculture Engineering, University of Illinois at Urbana-Champaign, 1997.

[2] Billingsley, J. and Schoenfisch, M. " Vision-Guidance of Agricultural Vehicles", Autonomous Robots, 2, pp. 65-76, 1995.

[3] Reid, J. F., "Precision Guidance of Agricultural Vehicles", SME Meeting, Sapporo, Japan. UILU-ENG-7031,1998.

[4] O Conner, M., Bell, T., Elkaim, G. and Parkingson, B., "Automatic Steering of Farm Vehicles Using GPS", in Proceedings of the 3rd International Conference on Precision Agriculture, June 1996.

[5] G. Elkaim, M. OConner, T. Bell and B. Parkinson, "System Identification and Robust Control of Farm Vehicles Using CDGPS", IONGPS-97, Kansas City, MO, USA, Vol 2:1415-1424, 1997.

[6] Thuilot B, Cariou C, Martinet P, and Berducat M, "Automatic guidance of a farm tractor relying on a single CP-DGPS", Autonomous robots , 13(1): 87-104,2002

[7] L. Cordesses, P. Martinet, B. Thuilot and M. Berducat, "GPSbased Control of a Land Vehicle",16th International Symposium on Automation \& Robotics in Construction, IAARC99, Madrid, Spain, September 22-24, 1999

[8] J. Ackermann, "Robust control prevents car skidding", IEEE Control Systems Magazine, pages 23-31, June 1997

[9] I. Motte, H. Campion, "Control of sliding mobile robots : a slow manifold approach", MNTS 2000

[10] W. Leroquais, B. D’ Andrea-Novel, “ Vibrational control of wheeled mobile robots not satisfying ideal velocity constraints: the unicycle case ”, European Control Conference, July 1-4, Brussels,1997

[11] Y. L. Zhang, J. H. Chung, S. A. Velinsky, " Variable structure control of a differentially steered wheeled mobile robot", Journal of intelligent and Robotic Systems, 36:301-314,2003

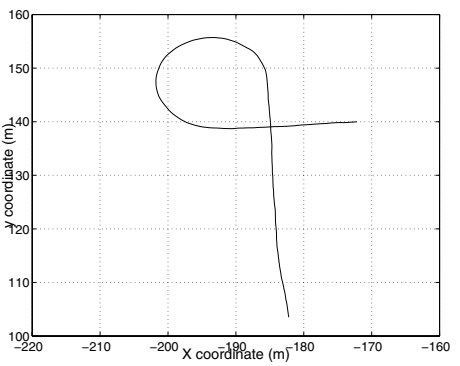

Fig. 7. Path \#2 to be followed

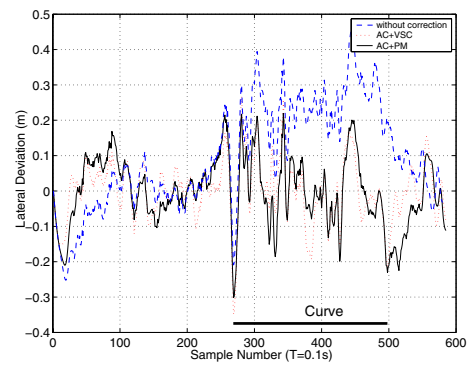

Fig. 8. Lateral deviation of path \#2 with real measurements

[12] H. Fang, R. Lenain, B. Thuilot, P. Martinet, "Sliding Mode Control of Automatic Guidance of Farm Vehicles in the Presence of Sliding ", The 4th International Symposium on Robotics and Automation, Queretaro, Mexico August 25-27, pp. 582-587, 2004

[13] M. L. Corradini and G. Orlando, "Experimental testing of a discretetime sliding mode controller for trajectory tracking of a wheeled mobile robot in the presence of skidding effects", Journal of robotic systems, 19(4), 177-188, 2002

[14] B. D’Andrea-Novel, G. Campion and G. Bastin. "Control of wheeled mobile robots not satisfying ideal constraints: a singular perturbation approach", International Journal of Robust Nonlinear Control, 5:243-267,1995.

[15] Lenain R. Thuilot B. Cariou C, Martinet P. "Adaptive control for car like vehicles guidance relying on RTK GPS: rejection of sliding effects in agricultural applications", In Proc. of the intern. Conf. On Robotics and Automation, Taipei, Sept, 2003.

[16] Z. P. Jiang and H. Nijmeijer, "A recursive technique for tracking control of nonholonomic systems in chained form",IEEE Transactions on Automatic Control, vol. 44, no. 2, 265-279, 1999.

[17] Kanellakopoulos, and P. Kokotovic, Nonlinear and Adaptive Control Design, John Wiley and Sons, ISBN: 0-471-12732-9, 1995.

[18] Y. P. Zhang, B. Fidan, P. A. Ioannou, " Backstepping control of linear time-varying systems with known and unknown parameters",IEEE Trans. Auto Contr., vol.48, pp. 1908-1925,2003

[19] Koshkouei, A. J. Zinober, A. S. I. “ Adaptive Backstepping Control of Nonlinear Systems with Unmatched Uncertainty", Proceedings of the 39th IEEE Conference on Decision and Control, Sydney, pp. 4765-4700, 2000

[20] B. Yao and M. Tomizuka, "Smooth robust adaptive sliding mode control of robot manipulators with guaranteed transient performance, Trans. of ASME, Journal of Dynamic Systems, Measurement and Control, vol. 118, no. 4, pp. 764-775, 1996.

[21] S. Sastry, M. Bodson, Adaptive Control: Stability, Convergence, and Robustness, Prentice-Hall, ISBN 0-13-004326-5, 1989

[22] Lenain R. Thuilot B. Cariou C, Martinet P. "Model Predictive Control of vehicle in presence of sliding Application to farm vehicles path tracking", To appear in ICRA05.

[23] H. Fang, "Automatic Guidance of Farm Vehicles in the Presence of Sliding ", scientific reports of LASMEA, http://srvlas.univbpclermont.fr/Control/index.html, 2004 